\title{
Strates
}

STRATES Matériaux pour la recherche en sciences sociales

$11 \mid 2004$

Jeune recherche, la vitalité d'un laboratoire

\section{Paroles d'habitants, discours sur le paysage : des modèles aux territoires}

L'évaluation des paysages du fleuve Loire, du Gerbier-de-Jonc à Nantes

\section{Sophie Bonin}

\section{(2) OpenEdition}

\section{Journals}

Édition électronique

URL : http://journals.openedition.org/strates/439

DOI : $10.4000 /$ strates.439

ISSN : $1777-5442$

Éditeur

Laboratoire Ladyss

Édition imprimée

Date de publication : 1 janvier 2004

ISSN : 0768-8067

\section{Référence électronique}

Sophie Bonin, «Paroles d'habitants, discours sur le paysage : des modèles aux territoires », Strates [En ligne], 11 | 2004, mis en ligne le 14 janvier 2005, consulté le 10 décembre 2020. URL : http:// journals.openedition.org/strates/439; DOI : https://doi.org/10.4000/strates.439

Ce document a été généré automatiquement le 10 décembre 2020.

Tous droits réservés 


\title{
Paroles d'habitants, discours sur le paysage : des modèles aux territoires
}

\author{
L'évaluation des paysages du fleuve Loire, du Gerbier-de-Jonc à Nantes
}

\section{Sophie Bonin}

1 Thèse de géographie soutenue à l'université de Paris I, le 8 janvier 2002, sous la direction d'Yves Luginbühl, directeur de recherche au laboratoire Ladyss/Cnrs (500 p.)

La plupart des fleuves ont généré des paysages importants pour l'homme, tant en termes d'occupation, de peuplement que d'aménagement de l'espace. De façon plus particulière, la Loire a en France largement participé à la construction de représentations du paysage, et à l'histoire même du concept de paysage. Et depuis peu, comme beaucoup de motifs naturels en Europe, elle fait l'objet de discours sur la séparation d'avec l'homme, ce qui pose en corrélation des problèmes de gestion, et simplement d'entretien. La Loire se présente, aujourd'hui, comme un terrain privilégié d'observation d'une nature complètement anthropisée et en même temps "sauvage » dans ses possibles excès hydrologiques et dans ses richesses écologiques.

Des sources à Nantes se retrouve l'idée d'une certaine "déprise " des sociétés riveraines sur l'espace fluvial. D'une part beaucoup d'activités locales liées à l'eau, qui représentaient des fonctions perçues à l'échelle individuelle jusque dans les années soixante, ont cessé. D'autre part le changement de mode de vie, plus rapide et mobile, et l'occupation de l'espace rural par des populations à dominante urbaine, conduisent à moins d'enracinement. Ces deux processus socio-économiques, en interaction, ont développé l'idée d'une fonction visuelle des cours d'eau : d'un fleuve décor, à préserver, à gérer en fonction de son aspect visuel et selon les modèles paysagers dominants dans la société, c'est-à-dire à gérer d'en haut. Ainsi de nombreux projets d'aménagement sont décidés pour la Loire à l'échelon national (le Plan Loire Grandeur Nature depuis 1994) et européen (le programme LIFE Loire-Nature), voire mondial puisqu'une partie de la Loire est, depuis décembre 2000, classée Patrimoine mondial de l'humanité par l'Unesco, au titre des paysages culturels. 
4 La recherche part d'une volonté d'interroger cette tendance vers un paysage visuel : il s'agit donc de préciser les modèles dominants à l'œuvre sur la Loire, tels qu'on les envisage de façon maintenant classique dans l'histoire des paysages vus, et c'est l'objet de la première partie. Il faut ensuite saisir quel est leur ancrage dans la matérialité des territoires et quelle est leur place dans les sociétés riveraines, ce qui est développé dans la deuxième partie. En bref, le ou les modèles paysagers de la Loire, construits par une culture nationale et pluri-séculaire, sont-ils modèles au sens de représentations simplifiées d'un système, et qui ont donc un sens heuristique pour les décisions en matière de gestion, ou ne sont-ils que modèles au sens de ce qui sert d'objet d'imitation, de référence, sans aucun souci de la dimension vivante du paysage?

5 La première partie se consacre donc à préciser les modèles paysagers dominants, à travers deux mouvements habituellement retenus dans les recherches sur la construction culturelle du paysage au sens de représentation. Ce sont d'abord les descriptions scientifiques, et un corpus de textes géographiques a été choisi, surtout depuis le XIX ${ }^{\mathrm{e}}$ siècle, quant à la place des fleuves, et en particulier de la Loire dans des manuels de géographie de la France. Ce sont ensuite des descriptions artistiques, à travers la peinture, et surtout la littérature, avec un corpus de citations littéraires très utilisées aujourd'hui. Le choix de discours écrits permet la mise en œuvre d'une méthode d'analyse de contenu qui comporte des étapes quantitatives et qualitatives, manuelle (thématique) et automatisée (lexicométrique). Cela conduit à la description de modèles physionomiques, ensembles de motifs relevés, en termes de formes, d'objets vus; et de modèles idéologiques, en termes de valeurs sous-jacentes, ou clairement attribuées au fleuve. La mise en évidence des modèles physionomiques n'a pas d'autre objectif qu'une meilleure connaissance des paysages et un éventuel cadre pour les projets d'aménagement, une source d'inspiration aussi. Mais celle des modèles idéologiques développe la compréhension des nombreux débats qui animent l'aménagement du territoire : à partir du moment où il $\mathrm{y}$ a des choix à faire, où des opinions sont en jeu, ce sont les valeurs que l'on accorde au fleuve qui animent les prises de position.

6 Mais en travaillant sur ce type de sources, de représentations très courantes dans l'ensemble de la société, on s'est placé d'emblée à une échelle globale: au fil de l'exploration des motifs mis en valeur, de nombreux décalages avec la matérialité des espaces sont apparus. Finalement l'idée est venue que la modélisation elle-même, en s'éloignant, en abstrayant les territoires, serait peu opérationnelle pour explorer les préférences paysagères, celles qui sont éprouvées et décrites à une échelle locale, ancrées sur un site. Aussi la deuxième partie de la recherche développe l'étude des représentations localisées, avec un choix de sources parmi divers groupes témoins du paysage du fleuve selon des distances différentes :

7 - les administrations et gestionnaires des territoires à travers les études paysagères telles qu'elles sont conçues depuis le début des années soixante-dix. Celles-ci représentent la distanciation professionnelle au paysage : les regards des architectes, des géographes, des ingénieurs, traduisent l'effort scientifique d'intégrer le paysage aux politiques d'aménagement. L'inventaire des études paysagères qui a été réalisé se révèle assez déficient en matière d'analyse des représentations, de prise en compte de la reconnaissance sociale, c'est-à-dire finalement d'études dans un autre sens que celui de simple décor. En revanche, elles témoignent des représentations que les paysagistes ont du fleuve. Cet inventaire sert donc surtout de source à l'étude des préférences 
paysagères, d'autant que le groupe que constitue ainsi les paysagistes avec les administrations commanditaires a un rôle sur les projets d'aménagement et sur l'intégration des grands ouvrages ;

8 - les touristes à travers les descriptions de la Loire dans une collection de guides touristiques, les Guides Bleus, qui offre un éventail de descriptions localisées de 1856 à nos jours. Le tourisme est une activité rituelle, très importante en bord de Loire, et qui occupe une grande place dans l'aspect des pays ligériens. Les auteurs des guides sont pris comme des porte-parole de cette activité, qui influencent les préférences paysagères par le biais des sites dont ils font la publicité. Pour explorer le système de représentations à l'œuvre, une méthode d'analyse des guides touristiques a été mise en place. Il s'est avéré aussi que se fixer sur un motif du paysage (un éco-symbole) fait du guide une source particulièrement utile pour analyser la spatialité et la temporalité des représentations qui sont véhiculées ;

9 - enfin les habitants, ceux qui vivent dans les communes riveraines de la Loire, à travers des enquêtes dans cinq communes, choisies dans cinq secteurs différents du fleuve : Brives-Charensac (Haute-Loire), Saint-Victor-sur-Loire (Loire, sur le plan d'eau du barrage de Grangent), Decize (Nièvre, sur une île), Chouzé-sur-Loire (Indre-et-Loire, face à la centrale nucléaire d'Avoine-Chinon), Saint-Florent-le-Vieil (Maine-et-Loire, dans un des paysages les plus "artialisés» du fleuve, et les plus conservés). Ils représentent la catégorie la moins explorée dans les approches classiques du paysage. Aussi sont-ils analysés comme un point d'aboutissement de la recherche : c'est grâce à la mise en place de modèles de représentations dans les autres groupes que l'on a cherché à caractériser les représentations des habitants, et à lier leurs préférences paysagères selon leurs discours et leurs pratiques. Ainsi l'analyse des catégories précédentes permet-elle d'explorer les discours des habitants sur le paysage, et de mettre en évidence l'existence d'un paysage de Loire original, du dedans du fleuve, chez les habitants.

10 Les discours de ces trois groupes, où peut s'ancrer l'expression locale des préférences paysagères, construisent une nouvelle démarche d'évaluation des paysages : c'est ce qui est éprouvé le long de la Loire, des sources à Nantes. Pour confirmer cette épreuve sur le terrain, on a aussi pris en compte les indicateurs du peuplement et de l'occupation du sol des communes riveraines, celles qui touchent le ruban d'un kilomètre de part et d'autre du lit mineur, par rapport aux départements traversés.

11 Finalement, on présente donc, selon les départements, l'évolution de la place de la Loire dans les communes riveraines de la source à l'estuaire, pour tenter une géographie des préférences paysagères: la réalité morphologique, physique et humaine, des territoires, celle de la géographie régionale, est mise en rapport avec ce que l'on appelle couramment le "paysage vu » et le «paysage vécu ».

12 On est ainsi passé à une échelle locale, qui offre finalement une autre approche du concept de paysage, par le bas, dont les résultats sont des représentations de nature différente. L'importance du fleuve comme facteur d'animation sociale, et comme support de pratiques de nature, est mise en évidence. Les pratiques douces ne sont pas apparues comme secondaires : la plus partagée, et que notre recherche tend à valoriser, est l'observation, le contact pluri-sensoriel qui lui est lié. Si modèle local il $\mathrm{y} a$, auprès des riverains, il s'agit d'une Loire sauvage au sens de vivante, non mécanique, mais à laquelle l'homme participe et dont il est responsable. La mise en œuvre d'une méthodologie pour appréhender cette échelle locale aboutit à tenter une géographie 
des préférences paysagères qui pourrait aider les aménageurs dans leurs démarches de gestion ou leurs projets touchant aux paysages. Les enquêtes réalisées, hormis celle qui le fut dans la commune riveraine d'un lac de barrage, témoignent d'une grande continuité dans une relation forte au fleuve, y compris chez les jeunes : les modalités et les fonctions de ce rapport ont changé, mais la connaissance intime et l'attachement des riverains sont très présents. Le paysage de Loire des riverains change donc plutôt moins que pour les autres catégories sociales selon la sectorisation classique du fleuve, de l'amont vers l'aval (Loire supérieure, moyenne et basse). Il change en revanche selon la configuration des rives, c'est-à-dire l'accessibilité, selon le courant de l'eau, vif ou mort, et selon la localisation du centre urbanisé, elle-même liée au rôle historique du fleuve pour ses habitants. C'est pourquoi les habitants des communes proches d'un grand barrage, selon notre étude, ont un rapport très perturbé voire annulé avec le cours d'eau. En revanche, ceux à proximité d'une centrale nucléaire ont cette relation intime avec la Loire qui existe un peu partout, presque dès les sources.

L'analyse lexicométrique comparative des paroles d'habitants par rapport aux citations littéraires montre, de façon attendue mais nette, que les premiers sont plus fidèles à la matérialité des territoires que les seconds : cela a pu être testé à propos de la présence du frêne par exemple. Les paroles d'habitants permettent enfin de prendre en compte la dimension tactile des paysages : ce qui ne se voit pas mais se sent, se devine, et qui est partie intégrante de l'image, visuelle, qui est finalement formée, et des préférences pour tel ou tel lieu. L'exemple de la qualité de l'eau, thème très sensible aux riverains mais absent de tous les autres modèles paysagers, en donne la mesure.

La prise en compte de ce qu'est le paysage pour les riverains ouvre donc un champ encore peu exploré pour l'évaluation des paysages : c'est un retour qui est proposé vers une plus grande attention au pays, autant en termes de matérialité que de vécu.

\section{INDEX}

Mots-clés : Paysage 